\title{
Politikwissenschaft, Humanismus und politische Anthropologie. Plädoyer für eine Renaissance klassischen politischen Denkens
}

\section{Mario Wintersteiger}

FB Politikwissenschaft und Soziologie, Universität Salzburg / Institut für Politikwissenschaft, Universität Innsbruck mario.wintersteiger@sbg.ac.at

\section{Zusammenfassung}

Der Beitrag zeigt, wie eine politiktheoretische Renaissance (im Sinne einer Reintegration klassischer, humanistischer Ansätze) dazu beitragen könnte, die zeitgenössische Politikwissenschaft von ihren blinden Flecken zu befreien. Ausgehend von einer Diagnose verbreiteter Mangelerscheinungen wird versucht, die Gräben zwischen natur- und kulturwissenschaftlichen Perspektiven zu überbrücken. Das Feld der politischen Anthropologie, das die Schnittstelle zwischen klassischer politischer Philosophie und Naturforschung darstellt, gilt als Fundament für die Brücke, auf der sich Humanismus und politikwissenschaftlicher Mainstream wieder annähern könnten. Eine besondere Rolle spielen jene Ansätze der antiken Philosophie, die Natur und Kultur des Menschen gleichermaßen thematisieren. Deren Anthropologie erweist sich als anschlussfähig an einen zeitgenössischen Wissensstand.

\section{Schlüsselwörter}

Politische Anthropologie, Politische Philosophie, Humanismus, Antike, Renaissance, Ideologiekritik

\section{Political science, humanism and political anthropology. A pleading for a renaissance of classical political thought}

\begin{abstract}
This contribution demonstrates how a renaissance in political theory (in the sense of a reintegration of classical humanistic approaches) could contribute to freeing contemporary political science from its blind spots. Starting with a diagnosis of widespread shortcomings, it tries to bridge the gap between natural sciences and cultural studies. The field of political anthropology, which is the interface between classical political philosophy and natural science, is providing the base for the bridge that enables humanism and the political science mainstream to meet again. Ancient philosophical approaches dealing with the nature and culture of men alike play a special role in this. Their anthropology turns out to be able to catch up with contemporary knowledge.
\end{abstract}

\section{Keywords}

Political Anthropology, Political Philosophy, Humanism, Antiquity, Renaissance, Critique of Ideology

The author has declared that no competing interests exist. 


\section{Einleitende Bemerkungen}

Wenn hier einer humanistischen Fundierung der Politikwissenschaft das Wort geredet wird, so heißt das nicht, dass die Forschung auf einen einseitigen Moralismus eingeschworen werden soll. ${ }^{1}$ Es geht auch nicht darum, einer ästhetischen Vorliebe zu frönen. Und es ist freilich kein rein antiquarisches Interesse, dem dieses Plädoyer entspringt. Ausgangspunkt ist vielmehr die Einsicht in das Ausmaß, in dem das kulturelle Erbe der Antike so innovative Zeitalter wie jenes der Renaissance beflügelt hat (vgl. darüber Rüstow 1963, 377-392). Der entscheidende Punkt ist hierbei, dass sich die damals forcierte Anlehnung an die klassischen, antiken Quellen befreiend und erhellend ausgewirkt hat (vgl. ebd., 390 f.). Dies führt uns zu der grundsätzlichen Überlegung, ob nicht humanistischer Geist auch heute noch eine ähnlich befruchtende Rolle spielen könnte, wenn es nur gelänge, ihn „in seiner frühen, renaissancehaften Kühnheit wiederherzustellen" (Paglia 1993, II3).

Spricht man - im hier gebrauchten Sinn des Wortes - von „Humanismus“, so wird damit ein klassischer Ansatz der Bildung bezeichnet, der seine Maßstäbe insbesondere aus dem Erbe der griechisch-römischen Antike schöpft (vgl. Jaeger 1960, IO5 ff.; Münkler 1993, 553 ff.). ${ }^{2}$ Unter seinem Zeichen vollzog sich während der Renaissance eine Wende hin zu einer neuen Leitdisziplin (der Historiographie! $)^{3}$ sowie zu einer freieren praktischen Philosophie - all dies in bewusster Abkehr von "scholastischen“ Denkmustern (vgl. ebd., 553-556 u. 56I), die nunmehr als weltfremd und pedantisch galten (vgl. dazu Schmidinger 1992, I335-1338). Die Bezeichnung ,scholastisch' wird auch gegenwärtig noch gebraucht, um hemmende Faktoren in den Wissenschaften zu benennen; so sieht etwa Lawrence M. Mead (2010) eine neuartige Form von „scholasticism“ in der heutigen Politikwissenschaft sich ausbreiten: Der dortige Mainstream - so die Kritik - habe in methodologischer Hinsicht zunehmend rigidere Züge angenommen und drohe dadurch mittlerweile den politologischen Wissenshorizont zu verengen (vgl. ebd., 453 f.).

Man sollte diese Mahnung nicht ignorieren. Scholastische Phänomene wie die von Mead genannte methodische Überspezialisierung und der daraus resultierende Wissens- und Relevanzschwund (vgl. darüber ebd., 453 f.) sind auch in der Politikwissenschaft des deutschsprachigen Raumes nicht unbekannt: So lässt sich feststel-

I Man beachte den Unterschied zwischen Humanismus und Philanthropie (vgl. dazu Babbitt 1956, 4 ff.).

2 Grundsätzlich könnte eine humanistische Haltung aber auch von anderen Traditionsbeständen als den griechisch-römischen ausgehen, etwa von asiatischen Quellen (vgl. dazu die Überlegungen zu einem „neuen Humanismus“ und der sog. „Zweiten Renaissance“ bei Eliade I973, I5-26; 75-77).

3 Damit einher ging auch ein Fokus auf zyklisches Geschehen im Geschichtsverlauf (vgl. Skinner 2000, Io9 f.). len, dass unserem Fach immer mehr sein Mittelpunkt abhanden kommt - was sich nachteilig auf den Einfallsreichtum und auf die Fähigkeit, öffentlich im "großen“ Maßstab mitzudiskutieren und zu gestalten, auswirkt (vgl. Straßenberger/Münkler 2007, 54 f.). Am wohl deutlichsten ablesen lässt sich diese Entwicklung an der zunehmenden Verdrängung der politisch-philosophischen und ideengeschichtlichen Bildung aus den politikwissenschaftlichen Curricula (vgl. darüber Buchstein/Fietz 2007). Vor dem Hintergrund dieser Vernachlässigung der "praktischen" Wissenschaft ${ }^{4}$ soll der vorliegende Beitrag auf politisch-anthropologische Grundfragen fokussieren und aufzeigen, dass sich die Politologie durch eine stärkere Reintegration klassischer, humanistischer Ansätze von manchen blinden Flecken befreien könnte. Er ist somit auch als Plädoyer für eine Renaissance der Politischen Theorie und Ideengeschichte als dem „Gravitationszentrum“ (Straßenberger/Münkler 2007, 54) der Gesamtdisziplin zu verstehen. Es mag manchen vielleicht paradox erscheinen, doch erweist sich gerade diese oft als praxisfern gescholtene geisteswissenschaftliche Bildung als eine höchst praxisrelevante; das gilt übrigens nicht allein für die Wissenschaften selbst, ihre Bedeutung zeigt sich zudem in demokratiepolitischer Hinsicht (vgl. dazu Strauss I995, 4 f. u. IO) und - neueren Untersuchungen zufolge - auch im heutigen Arbeitsleben (vgl. Stross 2017).

\section{Die Diagnose - Verengungen und Klüfte in der Politikwissenschaft}

In einem seiner Essays schrieb Irving Babbitt (1956, I9 ff.), es sei der Renaissance darum gegangen, den humanistischen Geist aus seiner Überlagerung durch die Theologie zu befreien - später sei aber die neue Aufgabe hinzugekommen, die so gewonnene Freiheit auch gegenüber der Domäne der Physik zu behaupten. Will man die gegenwärtige Lage diagnostizieren, so erweist sich dieser Gedanke noch als recht fruchtbar. Mead (2010, 453) führt den "scholasticism“ in der Politikwissenschaft auf eine allzu strenge Anwendung quasi-naturwissenschaftlicher Methoden zurück - ein Urteil, das hier unbedingt noch einer gewissen Differenzierung bzw. Präzisierung bedarf. Was uns als problematisch erscheint, sind nämlich nicht die Anleihen aus den Naturwissenschaften per se - insofern ist es wichtig, sich daran zu erinnern, dass ein Humanismus (im genuinen Wortsinn) diese Wissensbereiche gerade nicht ausblenden darf

4 Gemeint ist hier der aristotelische Praxisbegriff (vgl. dazu Ottmann 2005, I6-20). Man könnte daher auch von „prudentia“ sprechen - im Unterschied zur rein theoretischen „scientia“ (vgl. darüber Kriele I979, I7-2I).

5 Diese außerwissenschaftlichen Aspekte können hier nicht näher ausgeführt werden, wären jedoch gewiss eine eigene Abhandlung wert. 
(vgl. Brockman 2004, 8 f.). Was dagegen zu kritisieren ist, ist dreierlei: Erstens ahmen manche Sozialwissenschafter bloß nach, „was sie für die Methode der Naturwissenschaften halten [Hervorhebung des Verfassers!]" (Voegelin 1959, 369) - wohl nicht selten, ohne sich der dortigen wissenschaftstheoretischen Debatten ausreichend bewusst zu sein. ${ }^{6}$ Zweitens - und darauf haben insbesondere lebenswissenschaftlich versierte Autoren hingewiesen - lehnen sich viele zu eng an das (für sozialwissenschaftliche Zwecke weniger geeignete!) Vorbild der Physik an (vgl. Arnhart 20IO, 39; Masters I989, I3I u. 235). Drittens schließlich haben strenge Positivisten die - etwa von Eric Voegelin (2004, 2I ff.) beschriebene Neigung, alle Phänomene, die sich nicht quantifizieren lassen, als wissenschaftlich „irrelevant" abzutun. ${ }^{7}$ Erst die durch diese drei Punkte umrissene Haltung scheint uns für die von Mead (2010, 459) diagnostizierte Verengung der Politikwissenschaft verantwortlich zu sein: „The scholastic trends reflect an idea that political science, and social science generally, should be modeled on the physical sciences."

Der Mangel an humanistischer Perspektive, den diese neue Scholastik aufweist, wirkt sich in mehrfacher Hinsicht nachteilig aus: Nicht nur fördert er die inhaltliche Zersplitterung innerhalb der Disziplin (vgl. ebd., $455 \mathrm{ff}$.; Arnhart 20IO, 26), er vertieft auch die Kluft zwischen den verschiedenen wissenschaftlichen Zugangsweisen (vgl. ebd.). Die Folge scheint uns eine Verkürzung der Perspektiven zu sein - und zwar auf beiden Seiten des methodologischen Grabens: Wie Voegelin $(2004,22)$ zeigt, verlieren jene, die ausschließlich quasi-naturwissenschaftliche und quantifizierende Verfahren akzeptieren, eine Fülle politisch bedeutsamer Phänomene aus den Augen; dieser blinde Fleck kann - wie in jüngster Zeit wieder vermehrt festgestellt wird - auch bedenkliche gesellschaftliche Folgen haben (vgl. Muller 2018). Blickt man hingegen auf die andere Seite des Grabens, so ist mit Roger D. Masters (1989, xi) festzuhalten, dass der philosophisch inspirierte Zweig politischen Denkens mittlerweile dazu neigt, wissenschaftliche Einsichten in die Phänomene der Natur zu ignorieren - darin übrigens völlig konträr zu den antiken Klassikern, für die Natur eine zentrale Kategorie darstellte. All das muss früher oder später unweigerlich zu blinden Flecken - im Sinne einer „Lebens-, Geschichts- und Soziologieblindheit" (Röpke I979, IO8) - führen: Die Warnung, wonach ein rein mathematisches Paradigma die Gefahr mit sich bringt, die

6 Bemerkenswert ist das Ausmaß, in dem bestimmte Zweige der Naturwissenschaften die moderne Wissenschaftstheorie selbstkritisch reflektiert haben (vgl. hierzu und zu den Implikationen aus historiographischer Sicht Lukacs I994, 272-315).

7 Das hat nicht nur innerwissenschaftliche, sondern zunehmend auch breitere politische Implikationen. Neuere kritische Untersuchungen hierzu zeigen die negativen sozialen Auswirkungen, welche die einseitige Fixierung auf quantitative Daten mit sich bringt (vgl. z. B. Muller 20I8).
Sozialwissenschaften realitätsfern werden zu lassen (vgl. Mead 20I0, 453 f.), ist daher ebenso berechtigt wie die gleichlautende Mahnung an die Adresse mancher rein "kulturalistischer" Ansätze (vgl. dazu Illies 2009, I96 f.). Da der Mensch sowohl über natürliche als auch über kulturelle Prägungen geformt wird (vgl. dazu Arnhart 20IO), scheinen natur- und kulturwissenschaftliche Perspektiven jeweils ihren spezifischen Beitrag leisten zu können: Politologie könnte daher - wie Larry Arnhart (20IO, 36) es ausdrückt - „both a natural science and a humanistic art" sein. Wie wir aber schon gesehen haben, ist hierfür vielfach kein angemessenes Verständnis mehr gegeben, wodurch auch die Balance, die - nebenbei bemerkt - ein Merkmal der (aristotelischen) Klassik darstellte (vgl. Bloom I99I, 296), abhanden gekommen ist.

Will man die hieraus resultierenden Mangelerscheinungen, wie sie innerhalb der zeitgenössischen Politikwissenschaft verbreitet sind, thesenartig zusammenfassen, so ergibt sich folgendes Bild: (i) Dem von Mead (2010, 454) bedauerten „scholasticism“ mangelt es an Offenheit für qualitative Verfahren und somit an humanistischer Orientierung - ein Manko, das ja auch schon der historischen Scholastik vorgeworfen wurde (vgl. Schmidinger 1992, I336 f.). (ii) Ohne ein deutlich stärkeres „Gravitationszentrum“ (Straßenberger/Münkler 2007, 54), wie es politische Theoretiker als fächerübergreifende Klammer fordern (vgl. ebd.), werden die oben erwähnten methodologischen Gräben innerhalb der Disziplin vermutlich nicht zu überbrücken sein. (iii) Gelingt es nicht, die bereits angesprochene Balance zu finden, so wird die Politikwissenschaft tendenziell den (einen oder anderen) Aspekt der politischen Realität ausblenden. Ausgehend von dieser mehrschichtigen Diagnose soll nun exemplarisch erörtert werden, wie man mittels einer (angemessenen) Dosis klassisch inspirierten politischen Denkens zu einer breiter aufgestellten und auch realitätsnäheren Politologie gelangen könnte.

\section{Ein Therapievorschlag - Humanismus und Anthropologie als Brücken}

Ist man zu dem obigen Befund gekommen, so wird man sich nur schwerlich der Aufforderung entziehen können, die Empfehlung eines Heilmittels etwas näher zu erläutern. Ebenso thesenartig formuliert wie die Kritikpunkte zuvor folgt daher nun ein Therapievorschlag: Zunächst gilt es sich in methodischer Hinsicht offen und sachbezogen zu orientieren (vgl. dazu Voegelin 2004, 22 f.). Wie Voegelin schreibt, darf sich politische Theorie keiner (wie auch immer gearteten) methodologischen Einseitigkeit beugen (vgl. ebd., 22-25). Danach muss man sich darum bemühen, mittels theoretischer wie ideengeschichtlicher Anstrengungen einen „integrativen Zusammenhalt" (Straßenberger/Münkler 2007, 54) 
zu schaffen. Die Aufgabe, die es hierbei zu übernehmen gilt, hat Gerhard Göhler $(2007,87)$ als die Arbeit an einer „Ordnung der politischen Erfahrungen“ gut auf den Punkt gebracht. Das damit verbundene Anforderungsprofil ist freilich ein anspruchsvolles: Eine breite, auch humanistische Bildung - eine "liberal edcuation" (Arnhart 20IO, 26 u. 40) - anzustreben, scheint hierfür praktisch unerlässlich zu sein. Mitunter verspricht man sich von einer solchen nicht weniger als eine bessere Verankerung der Politologie im weiten Feld der wissenschaftlichen Disziplinen (vgl. ebd., 26). Uns interessiert hierbei auch, wie dadurch die bereits erwähnte Kluft zwischen quasi-naturwissenschaftlicher und kultureller Betrachtungsweise verringert werden könnte. ${ }^{8}$

Die Tatsache, dass sich humanistische Forschung seit jeher vor allem für sprachliche Zeugnisse, historische Fakten und politisch-philosophische Ideen interessiert (vgl. Münkler 1993, 559-562), rückt sie freilich sehr nahe an die kulturwissenschaftlichen Hermeneutiken heran (vgl. hierzu Reckwitz 2004, 36 ff.). Das bedeutet jedoch keineswegs, dass sie sich anderen Wissensgebieten gegenüber verschließen darf. Während der Renaissance übrigens hat man dies keineswegs getan, sondern sich auch für Naturforschung interessiert (vgl. Brockman 2004, 8 f.). Ist die Arbeit an einer solch tragfähigen theoretischen Brücke für die heutige Politikwissenschaft eine intellektuelle Heraklesaufgabe, die inzwischen kaum mehr zu bewältigen ist? So herausfordernd sie auch sein mag, worüber wir immerhin bereits verfügen, ist eine Art Bauplan - man findet ihn im ideengeschichtlichen Fundus. In seinem Buch The Nature of Politics weist Masters (I989, xi f.) darauf hin, dass die klassisch-griechische Philosophie keine völlige Kluft zwischen Politik und Natur kannte, weil die Denkkategorie einer "human nature" einen festen Anker für das politische Denken darstellte. In einer davon inspirierten Manier schlägt er vor, die Theorie der Politik mit dem Wissen der Verhaltensforschung und anderer Lebenswissenschaften anzureichern, um so philosophische und naturwissenschaftliche Zugänge wieder miteinander zu versöhnen (vgl. ebd., xii f.). Dieses Modell scheint eine Sonderform dessen zu sein, was man mit Henning Ottmann (2005) als "neoklassische politische Philosophie“ bezeichnen könnte. Es bekennt sich zu den politischen Klassikern, behält die philosophische Stoßrichtung bei, achtet dabei jedoch darauf, den Anschluss an die „hard' sciences" nicht zu verpassen (vgl. Masters I989, I79 f.). Wie auch immer man diesen Ansatz in allen Details beurteilen mag, was für uns hier interessant ist, ist erstens, dass es prinzipiell möglich ist, Brücken zwischen den Paradigmen zu bauen, und zweitens, dass es hierbei

8 Die Grundidee der "liberal education“ geht offensichtlich von einer derartigen Möglichkeit aus (vgl. dazu Arnhart 20I0, 26 u. 40; 2006). offenbar gerade der humanistische Rückgriff ist, der es erst erlaubt, die Kluft zu überbrücken.

Daraus lässt sich wohl für den von uns skizzierten Therapievorschlag lernen; doch zunächst gilt es noch davor zu warnen, dieses politikwissenschaftliche Programm als ein politisch-ideologisches Projekt misszuverstehen: ${ }^{9}$ Wie schon Voegelin (2004, I4) in seinem Werk Die Neue Wissenschaft der Politik schreibt, geht es hierbei nämlich "nicht um die Wiederaufnahme platonisch-aristotelischer Doktrinen“, es geht keineswegs um die konkreten politischen Inhalte solcher Klassiker, sondern allein um deren politiktheoretischen Horizont (vgl. ebd.). Diesen wiederum sieht er durch eine Grundstruktur geprägt, der gemäß jene politikwissenschaftlichen Fragen, die es „empirisch und kritisch“ (ebd., 28) zu behandeln gilt, vor dem Hintergrund anthropologischer Grundlagen beantworten werden müssen (vgl. ebd., 28 f.). Mit letzter Bemerkung ist nun das wahrscheinlich aussichtsreichste Fundament für die humanistische Brücke, um die es hier geht, markiert: Wir sind gegenwärtig immerhin Zeugen einer gewissen „Renaissance der politischen Anthropologie“ (Jörke 2005, 9) und haben zudem ein lebendiges historisch-anthropologisches Forschungsfeld vor uns (vgl. dazu Tanner 2004), dessen Funde sich auswerten lassen. ${ }^{10}$ Damit aber scheint nun ein Schnittpunkt gefunden zu sein, von dem aus man arbeiten kann: Die politische Philosophie kann sich genau dort mit neuem Erfahrungswissen nähren. ${ }^{\text {II }}$ Der zeitgenössische Mainstream wiederum kann von dort aus einen Schritt auf die klassischen Perspektiven zugehen, da mit dem Fokus auf "humane Universalien“" (Jörke 2005, Io) wieder ein beiden Paradigmen gemeinsamer Boden erreicht ist (vgl. dazu Arnhart 20IO, 26 ff.; Masters 1989, xi ff.). Ein klingendes Motto für die dortigen Begegnungen gibt es bereits: „Erkenne dich selbst“ (Jaeger 1960, III), lautet der klassisch-humanistische Leitspruch, der nun in einer aktualisierter Form wieder ausgegeben wird (vgl. Masters 1989, xv u. 249). Seine politikwissenschaftliche Bedeutung wird in den nun folgenden Ausführungen über die Potentiale der politischen Anthropologie näher erörtert werden.

9 Konzepte zur Erneuerung antiker politischer Philosophie werden häufig verzerrt dargestellt; sie leiden mitunter unter ideologisch motivierten Anfeindungen oder haben sich den Versuchen politischer Vereinnahmung zu erwehren (vgl. darüber schon Voegelin 2004, I4).

IO Der Bereich der sog. „historischen Anthropologie“ ist zu vielfältig, als das er hier ausführlich dargestellt werden könnte. Die Palette umfasst naturwissenschaftliche Genealogien, aber auch kulturwissenschaftliche Interpretation historischer Artefakte (vgl. dazu Tanner 2004, 9-I3); für eine politische Anthropologie erweisen sich beide Zugänge als Fundgruben.

II Allgemein zur Rolle der Empirie für die politische Philosophie vgl. Göhler (2007, 85-90). 


\section{Einige Perspektiven der politischen Anthropologie und Ideologiekritik}

Schon Thukydides, der unter methodischen Gesichtspunkten als der frühe Pionier einer wissenschaftlichen Geschichtsdarstellung gesehen werden kann (vgl. dazu Ottmann 200Ia, I37 ff.), setzt in seiner Schrift Der Peloponnesische Krieg $(\mathrm{I}, 22)$ die Kategorie einer „menschlichen Natur" voraus, die über vergangene Erfahrungen feststellbar sei und zudem Schlüsse über zukünftige Ereignisse ermögliche (vgl. Thukydides 1970, 56; Ottmann 200Ia, I38). Seine Grundidee ist gar nicht so verschieden von jener der politischen Anthropologie, wonach "das Aufgabenspektrum der Politik durch die Zeiten hindurch [...] relativ konstant geblieben ist, weil sich die Probleme, um deren Lösung sich Politik bemüht, auf bestimmte relativ stabile Strukturen des Menschen zurückführen lassen“ (Birnbacher 2009, I80). Welche politikwissenschaftlichen Perspektiven eröffnet uns nun diese grundsätzliche Einsicht? Voegelin $(2004,75)$ sieht das "anthropologische Prinzip“ der platonischen Tradition als ein doppeltes an: Es ist demnach zugleich ein verstehendes und ein kritisches Hilfsmittel, das einerseits ermöglichen soll, Gemeinwesen von den konkreten Menschen her zu interpretieren, das aber andererseits auch einen Qualitätsmaßstab für politische Systeme anbieten will (vgl. ebd., 75 ff.).

Wie jedoch könnte hier eine zeitgemäße Adaptierung aussehen? Und welche Probleme müssten auf dem Weg dorthin gelöst werden? Was eine echte Herausforderung darstellt, ist der Umstand, dass der „Verweis auf die Natur des Menschen" (Illies 2009, 196) heute von vielen Sozialwissenschaftern äußerst ungern gesehen wird - häufig auch aus politischen Motiven heraus (vgl. ebd., I96 f.). Demgegenüber kann man aber zumindest zwei Dinge ins Feld führen: Erstens ist festzuhalten, dass sehr wohl eine beträchtliche Anzahl allgemeinmenschlicher, in der Natur verwurzelter „Universalien“ existiert (vgl. darüber Welsch 2006, I26-I38). Und zweitens gilt es zu bedenken, dass wohl - wie David J. Levy (I987, 3I) schreibt - solche anthropologischen Einsichten einen entscheidenden Faktor darstellen, der die politische Theorie davor bewahren kann, ideologisch verfälscht zu werden. Freilich muss man sich bei all dem immer bewusst bleiben, dass die "menschliche Natur" weder eindimensional aufgebaut (vgl. dazu Welsch 2006, I32 ff.), noch gänzlich starr ist (vgl. Masters I989, 245). Dennoch aber lassen sich auch "harte Universalien“ (Welsch 2006, I30) feststellen: Wie Wolfgang Welsch zeigt, sind dies organisch bedingte anthropologische Konstanten (vgl. ebd., I35-I38), aus denen sich wiederum menschliche „Bedürfnis-Universalien“ (ebd., I43) herauskristallisieren, die jede Kultur mit vergleichbaren Problemstellungen konfrontieren (vgl. ebd., I43 f.). Dieser Umstand und auch die Tatsache, dass natürliche Faktoren die Grund- struktur von Präferenzen vorformen können (vgl. dafür ebd., I3I-I34), dürfte klar machen, worin die Bedeutung einer wissenschaftlich fundierten Menschenkenntnis besteht. Ihr fällt keine geringere Aufgabe zu, als jene, dem politischen Denken eine realistische Basis zu verleihen: „Anthropologische Faktoren gehören [...] zu den Sachaspekten, die das zu bewältigende Problem konstituieren", bringt es Dieter Birnbacher (2009, 182) auf den Punkt und unterstreicht zudem, dass es ohne entsprechende Einsichten auch gar nicht möglich wäre, die künftig zu erwartende Wirkweise politischer Maßnahmen seriös zu beurteilen (vgl. ebd., I82 f.).

Modifiziert man das klassische „anthropologische Prinzip“ (Voegelin 2004, 75), indem man es auf zeitgemäßen Grundlagen neu aufstellt und dabei auch seine „Ideologieanfälligkeit“ (Schockenhoff I996, I9I) minimiert, so gewinnt man dadurch ein brauchbares Hilfsmittel, um gesellschaftliche Systeme zu verstehen, menschliche Handlungsweisen zu erklären und politische Ideen zu bewerten: ${ }^{12}$ Es kann wie gehabt zur Hermeneutik beitragen, indem es - ähnlich dem von Voegelin $(2004,75$ f.) vorgestellten platonischen Verfahren - die Gemeinwesen im Lichte prägender menschlicher Charakteristika liest. Darüber hinaus erlaubt es die Kenntnis der menschlichen Natur, politische Phänomene zu erklären - beispielsweise die Psychologie der Macht (vgl. dazu Ludwig 2002). Schließlich ist den anthropologischen Einsichten noch zu attestieren, dass sie - wie schon kurz angemerkt wurde - eine Schlüsselrolle bei der Arbeit an politischen Ideen spielen: Um dies zum Ausdruck zu bringen, vergleichen manche die politische Philosophie mit der angewandten Medizin, deren praktische Ausübung ebenfalls ein Wissen um den Menschen voraussetzt (vgl. McShea 1978, 658 f.; Kriele 1979, I8; Masters I989, xv). Woran hierbei gedacht wird, ist offenbar eine Art von politiktheoretischer Risikobewertung (vgl. ebd.). Und was damit konkret gemeint ist, wird gewiss klarer, wenn man sich verdeutlicht, wie die Wissenschaft „über Grenzen von Handlungsmöglichkeiten und über die Folgen von Handlungen und Normensystemen Aussagen macht" (Mohr 1987, 66). Spätestens hier wird auch das kritische Potential der Anthropologie, um das schon die Klassik wusste (vgl. Voegelin 2004, 75 ff.), offenbar. Ihre Erkenntnisse ermöglichen uns gewissermaßen den Blick durch eine ideologiekritische Linse: "Je spekulativer die Anthropologie, desto geschlossener und absolutistischer die Geschichtsphilosophie", stellt Ram Adhar Mall $(2000,3)$ fest - und hat dabei natürlich das historische Scheitern der Ideologien vor Augen

\footnotetext{
I2 Um diese Aufgabe seriös erfüllen zu können, darf man freilich nicht - wie bei politischen Ideologien und scholastischen Naturrechtslehren oft üblich - „beliebige Annahmen“ über die menschliche Natur zum Ausgangspunkt machen (vgl. dazu Schockenhoff I996, I90-I95.). Die Anthropologie darf sich nicht politisch manipulieren lassen, muss doch gerade die Politik in ihrem Lichte adäquat beurteilt werden können.
} 
(vgl. ebd., 2 ff.). Sein Plädoyer für eine realitätsnähere Anthropologie (vgl. ebd., I-6) scheint uns somit auch für die politische Philosophie unmittelbar relevant zu sein: Dieser nämlich geht es - mit Klaus von Beyme (2000, 49) gesprochen - um den „Abbau von Irrationalität im Bereich der Aussagen über das Sollen“.

Vor dem Hintergrund des so nur grob umrissenen Potentials einer anthropologisch fundierten Politikwissenschaft lässt sich nun gut aufzeigen, worin die diesbezügliche Bedeutung einer Renaissance klassisch-humanistischer Ansätze liegt. Ein Plädoyer dafür drängt sich insbesondere deshalb auf, weil die politisch-anthropologische Fundierung vielfach eine Schwachstelle zeitgenössischer Theorieansätze darstellt: Schon der neueren vertragstheoretischen Zugangsweise (à la John Rawls) wirft man mitunter vor, ein verkürztes Menschenbild zu skizzieren (vgl. Ankersmit 1996, 2 ff.; Bloom 199I, $300 \mathrm{ff}$.). In den Ideenkreisen des Neomarxismus und Postmodernismus aber stoßen anthropologische Argumentationslinien vielfach per se auf offene Ablehnung (vgl. darüber Jörke 2005, 48-56). Zur Problematik dieser Situation gehört auch, dass all jene, die sich um eine stärkere Integration der Anthropologie bemühen, noch einige weitere Fallstricke der politischen Theorie bzw. Philosophie vermeiden müssen: Bei Erklärungsversuchen hat man sich etwa davor zu hüten, einer anderen großen Einseitigkeit, nämlich dem (als politische Ideologie problematischen!) „Biologismus“ (vgl. darüber Wuketits 20IO, 93 f.) zu verfallen. Zudem gilt es zu vermeiden, wissenschaftstheoretisch unzulässige normative Schlussfolgerungen zu ziehen (vgl. dazu MacIntyre I959, 463 f. u. 467; Sutor 1984, 35 ff.); dass tatsächlich die Gefahr besteht, dass sich Fehler in die Argumentationslogik einschleichen, zeigt die Auseinandersetzung mit allzu abstrakten Naturrechtslehren, bei denen die Auffassung von der "Natur des Menschen" mitunter durch politische oder ethische Wünsche korrumpiert ist (vgl. dazu Schockenhoff 1996, 25-30); vermeiden kann man die Fallstricke, wenn man bedenkt, dass "theoretische“ Naturerkenntnis und "praktische Vernunft" nicht völlig deckungsgleich sind (vgl. ebd., I83). Summa summarum kommt die Arbeit an einer anthropologisch fundierten Politikwissenschaft wohl einer Fahrt zwischen Syclla und Charybdis gleich. In den nun folgenden, abschließenden Ausführungen soll exemplarisch gezeigt werden, inwiefern uns ideengeschichtliche, humanistische Impulse bei dieser anspruchsvollen Herausforderung helfen können.

\section{Von der antiken Anthropologie zu einer Renaissance in der Politikwissenschaft?}

Was die griechische Philosophie der Antike politischanthropologisch interessant macht, sind ihre Pionier- leistungen auf besagtem Gebiet: Als die ersten quasihumanistischen Denker kann man mit Ottmann (200Ia, 213) die Sophisten nennen. Diese waren es auch, die einen "Gegensatz zwischen physis und nomos, Natur und Gesetz" (ebd., 214) aufzeigten. Die damalige Grundproblematik leuchtet noch immer unmittelbar ein: „Die vermehrte Kenntnis anderer Länder und Sitten“, hebt Ottmann hervor, „stellt die Frage, was am Nomos natürlich, was an ihm künstlich ist." (ebd.) Die Thematik birgt heute noch so manchen Zankapfel. Es geht dabei auch darum, welche Aspekte mit natur- und welche nur mit kulturwissenschaftlichen Methoden einsichtig gemacht werden können. ${ }^{13}$ Über all dem dürfen aber nicht die Querverbindungen zwischen den beiden Domänen übersehen werden: Eine zeitgemäße Anthropologie muss davon ausgehen, dass die Natur den Menschen in die Kultur drängt, umgekehrt jedoch auch die Kultur des Menschen auf seine Natur einwirkt (vgl. dazu Arnhart 2006, 9; Welsch 2006, I32 ff.). Den zuerst genannten Punkt findet man schon in der Urform humanistischer Anthropologie, so etwa im "Mythos des Protagoras" (Ottmann 200Ia, 220): In der durch Platon (2009, 35-39; Ottmann 200Ia, 220 f.) tradierten Lehrerzählung wird das Wesen des Menschen gegenüber den anderen Lebewesen charakterisiert, indem auf seine herausragende "technische Intelligenz" (symbolisiert durch das Feuer des Prometheus) sowie auf seine politischen Ideen (im Mythos überbracht durch Hermes) verwiesen wird. Die Botschaft scheint hierbei klar zu sein und wird von Ottmann auf den Punkt gebracht: „Der Mensch ist von Natur aus ein Kulturwesen." (ebd., 22I) Trotz seines doch beträchtlichen Alters scheint dieser Grundgedanke unvermindert seine analytische Fruchtbarkeit bewahrt zu haben. Seine Wiederkehr in der Anthropologie jüngeren Datums bezeugt seine bleibende Aktualität. ${ }^{14}$

Daneben offenbaren bei näherer Betrachtung noch einige andere Linien der antiken politischen Philosophie großes Potential, Anschluss an einen neueren anthropologischen Wissensstand zu finden und im Gegenzug adaptierbare humanistische Perspektiven anzubieten: $\mathrm{Zu}$ nennen gäbe es hier beispielsweise die Epikureer und deren „Lehre von der Kulturentstehung“ (Müller 1983, 200). Für dieses Modell ist es - wie Reimar Müller zusammenfasst - charakteristisch, dass es den Menschen als ein Natur- und Kulturwesen ansieht, wobei natürliche Faktoren den Menschen im Zuge seiner Entwicklung dazu bringen, sich mit seiner Kultur eine „zweite Natur“ innerhalb der Möglichkeiten der ersten zu schaffen (vgl. ebd., 197-200). Bemerkenswert ist diesbezüglich auch, dass Kenner der Materie dem römischen Epikureer Lu-

I3 Das hat freilich auch Auswirkungen auf den Charakter des jeweils erreichbaren Wissens - ein Umstand, um den bereits die (aristotelische) Klassik wusste (vgl. dazu Ottmann 200Ib, I20 ff.).

I4 Hinweise auf diese Kontinuitäten finden sich bei Ottmann (200Ia, 220 f.) sowie bei Pöhlmann (I970, 299 f. u. 304 f.). 
krez attestieren, schon einige Einsichten der modernen Biologie vorweggenommen zu haben (vgl. Riedl 2003, I7 f.; Masters 1989, I49).

Somit zeigt sich das Bild einer doch überraschend großen Aktualisierbarkeit bestimmter Ansätze der antiken Philosophie. Damit ist nun jener Schnittpunkt zwischen humanistischem Erbe und naturwissenschaftlicher Erkenntnis eingekreist, von dem vorhin bereits die Rede war. Dass sich beide auf dem Gebiet der politischen Anthropologie begegnen können, deutet darauf hin, dass eine zeitgemäße Renaissance ideengeschichtlich tradierter Klassiker durchaus möglich ist. Ihr Potential liegt unter anderem darin, dass sie die Prototypen für einen Zugang anbieten, der Natur und Kultur des Menschen gleichermaßen berücksichtigt, also jene Einseitigkeit vermeidet, die viele zeitgenössische politische Modelle auf die eine oder andere Weise kennzeichnet. Wie aber kann heute noch eine politiktheoretische Brücke gebaut werden, die es erlaubt, natur- und kulturwissenschaftliche Perspektiven in eine fruchtbare Politikwissenschaft zu integrieren? Die philosophisch orientierte Seite hat dafür zu sorgen, dass ihre Grundlagen „empiriegesättigt“ (Göhler 2007, 85) bleiben. Zudem ist sicherzustellen, dass in ihr Fundament auch der Stand der naturwissenschaftlich ausgerichteten Forschung mit eingearbeitet wird, allerdings ohne dabei in deren spezifische Einseitigkeit zu verfallen (vgl. dazu Welsch 2006, I4I). Gelingt es so, den klassischen und humanistischen Perspektiven wieder breitere Akzeptanz innerhalb der Gesamtdisziplin zu verschaffen, könnte diese kleine Renaissance dazu beitragen, die zeitgenössische Politikwissenschaft von ihren erwähnten Verengungen und Klüften zu befreien.

\section{Literatur}

Ankersmit, Frank R. (1996), Aesthetic Politics: Political Philosophy Beyond Fact and Value, Stanford: Stanford University Press.

Arnhart, Larry (2010), Biopolitical science, in: Politics and the Life Sciences, Vol. 29(I), 24-27.

Arnhart, Larry (2006), Darwinian Liberal Education, in: Academic Questions, Vol. I9(4), 6-I8.

Babbitt, Irving (1956), Literature and the American College: Essays in Defense of the Humanities, Los Angeles-Chicago-New York: Gateway Editions.

Beyme, Klaus von (2000), Die politischen Theorien der Gegenwart: Eine Einführung, Wiesbaden: Westdeutscher Verlag.

Birnbacher, Dieter (2009), Was kann die Anthropologie zur Politik und ihrer theoretischen Fundierung beitragen? In: Jörke, Dirk/Bernd Ladwig (Hg.), Politische Anthropologie: Geschichte - Gegenwart - Möglichkeiten, Baden-Baden: Nomos, I79-194.
Bloom, Allan (199I), Giants and Dwarfs: Essays 1960I990, New York u. a.: Simon and Schuster.

Brockman, John (2004), Einführung: Die neuen Humanisten, in: Brockman, John (Hg.), Die neuen Humanisten: Wissenschaft an der Grenze, Berlin: Ullstein, 7-I8.

Buchstein, Hubertus/Stefan Fietz (2007), Vom Verschwinden bedroht? Politische Theorie und Ideengeschichte in der curricularen Reformfalle, in: Österreichische Zeitschrift für Politikwissenschaft, Vol. 36(I), 67-79.

Eliade, Mircea (1973), Die Sehnsucht nach dem Ursprung. Von den Quellen der Humanität, Wien: Europaverlag.

Göhler, Gerhard (2007), Theorie als Erfahrung: Über den Stellenwert von politischer Philosophie und Ideengeschichte für die Politikwissenschaft, in: Buchstein, Hubertus/Gerhard Göhler (Hg.), Politische Theorie und Politikwissenschaft, Wiesbaden: VS Verlag für Sozialwissenschaften, 80-IO4.

Illies, Christian (2009), Die Bedeutung von Anthropologie und Evolutionswissenschaften für die politische Philosophie, in: Jörke, Dirk/Bernd Ladwig(Hg.), Politische Anthropologie: Geschichte - Gegenwart - Möglichkeiten, Baden-Baden: Nomos, I95-229.

Jaeger, Werner (1960), Humanistische Reden und Vorträge, Berlin: de Gruyter.

Jörke, Dirk (2005), Politische Anthropologie: Eine Einführung, Wiesbaden: VS Verlag für Sozialwissenschaften.

Kriele, Martin (1979), Recht und praktische Vernunft, Göttingen: Vandenhoeck \& Ruprecht.

Levy, David J. (1987), Political Order: Philosophical Anthropology, Modernity, and the Challenge of Ideology, Baton Rouge-London: Louisiana State University Press.

Ludwig, Arnold M. (2002), King of the Mountain: The Nature of Political Leadership, Lexington: University Press of Kentucky.

Lukacs, John (1994), Historical Consciousness: The Remembered Past, New Brunswick: Transaction Publishers.

MacIntyre, Alasdair C. (1959), Hume on 'Is' and 'Ought', in: The Philosophical Review, Vol. 68(4), 45I-468.

Mall, Ram Adhar (2000), Mensch und Geschichte: Wider die Anthropozentrik, Darmstadt: Wissenschaftliche Buchgesellschaft.

Masters, Roger D. (1989), The Nature of Politics, New Haven-London: Yale University Press.

McShea, Robert J. (1978), Human Nature Theory and Political Philosophy, in: American Journal of Political Science, Vol. 22(3), 656-679.

Mead, Lawrence M. (2010), Scholasticism in Political Science, in: Perspectives on Politics, Vol. 8(2), 453-464.

Mohr, Hans (1987), Natur und Moral: Ethik in der Biologie, Darmstadt: Wissenschaftliche Buchgesellschaft.

Müller, Reimar (1983), Zu einem Entwicklungsprinzip 
der epikureischen Anthropologie, in: Philologus: Zeitschrift für klassische Philologie, Vol. I27(2), I87-206.

Münkler, Herfried (1993), Die politischen Ideen des $\mathrm{Hu}-$ manismus, in: Fetscher, Iring/Herfried Münkler (Hg.), Pipers Handbuch der Politischen Ideen, Bd. 2, München-Zürich: Piper, 553-6I3.

Muller, Jerry Z. (2018), The Tyranny of Metrics, Princeton-Oxford: Princeton University Press.

Ottmann, Henning (2005), Platon, Aristoteles und die neoklassische politische Philosophie der Gegenwart, Baden-Baden: Nomos.

Ottmann, Henning (200Ia), Geschichte des politischen Denkens, Bd. I/I: Die Griechen. Von Homer bis Sokrates, Stuttgart-Weimar: J. B. Metzler.

Ottmann, Henning (200Ib), Geschichte des politischen Denkens, Bd. I/2: Die Griechen. Von Platon bis zum Hellenismus, Stuttgart-Weimar: J. B. Metzler.

Paglia, Camille (1993), Der Krieg der Geschlechter: Sex, Kunst und Medienkultur, Berlin: Byblos.

Platon (2009), Protagoras: Griechisch/Deutsch, Stuttgart: Reclam.

Pöhlmann, Egert (1970), Der Mensch - das Mängelwesen? Zum Nachwirken antiker Anthropologie bei Arnold Gehlen, in: Wagner, Fritz (Hg.), Archiv für Kulturgeschichte, 52. Bd., Köln-Wien: Teubner, 297-312.

Reckwitz, Andreas (2004), Die Politik der Moderne aus kulturtheoretischer Perspektive: Vorpolitische Sinnhorizonte des Politischen, symbolische Antagonismen und das Regime der Gouvernementalität, in: Schwelling, Birgit (Hg.), Politikwissenschaft als Kulturwissenschaft: Theorien, Methoden, Problemstellungen, Wiesbaden: VS Verlag für Sozialwissenschaften, 33-56.

Riedl, Rupert (2003), Riedls Kulturgeschichte der Evolutionstheorie: Die Helden, ihre Irrungen und Einsichten, Berlin u. a.: Springer.

Röpke, Wilhelm (1979), Civitas Humana: Grundfragen der Gesellschafts- und Wirtschaftsreform, BernStuttgart: Haupt.

Rüstow, Alexander (1963), Ortsbestimmung der Gegenwart: Eine universalgeschichtliche Kulturkritik, 2. Bd.: Weg der Freiheit, Erlenbach-Zürich-Stuttgart: Rentsch.

Schmidinger, Heinrich M. (1992), Scholastik, in: Ritter, Joachim/Karlfried Gründer (Hg.), Historisches Wörterbuch der Philosophie, Bd. 8: R-Sc., Basel: Schwabe, I332-I342.

Schockenhoff, Eberhard (1996), Naturrecht und Menschenwürde: Universale Ethik in einer geschichtlichen Welt, Mainz: Matthias-Grünewald-Verlag.

Skinner, Quentin (2000), The foundations of modern political thought, $\mathrm{Bd}$. I: The Renaissance, Cambridge $\mathrm{u}$. a.: Cambridge University Press.

Straßenberger, Grit/Herfried Münkler (2007), Was das Fach zusammenhält: Die Bedeutung der Politischen Theo- rie und Ideengeschichte für die Politikwissenschaft, in: Buchstein, Hubertus/Gerhard Göhler (Hg.), Politische Theorie und Politikwissenschaft, Wiesbaden, VS Verlag für Sozialwissenschaften, 45-79.

Strauss, Leo (1995), Liberalism Ancient and Modern, Chicago-London: The University of Chicago Press.

Stross, Randall (2017), A Practical Education. Why Liberal Arts Majors Make Great Employees, Stanford: Redwood Press.

Sutor, Bernhard (1984), Neue Grundlegung der politischen Bildung, Bd. I: Politikbegriff und politische Anthropologie, Paderborn u. a.: Schöningh.

Tanner, Jakob (2004), Historische Anthropologie zur Einführung, Hamburg: Junius.

Thukydides (1970), Der Peloponnesische Krieg: Auswahl, Stuttgart: Reclam.

Voegelin, Eric (2004), Die Neue Wissenschaft der Politik: Eine Einführung, München: Wilhelm Fink Verlag.

Voegelin, Eric (1959), Diskussionsbereitschaft, in: Hunold, Albert (Hg.), Erziehung zur Freiheit, Erlenbach-Zürich-Stuttgart: Rentsch, 355-372.

Welsch, Wolfgang (2006), Über Besitz und Erwerb von Gemeinsamkeiten, in: Bickmann, Claudia/HermannJosef Scheidgen u. a. (Hg.), Tradition und Traditionsbruch zwischen Skepsis und Dogmatik: Interkulturelle philosophische Perspektiven, Amsterdam-New York: Rodopi, II3-I45.

Wuketits, Franz M. (20I0), Die Entdeckung des Verhaltens: Eine Geschichte der Verhaltensforschung, Darmstadt: Wissenschaftliche Buchgesellschaft.

\section{Autor}

Dr. Mario Wintersteiger ist wissenschaftlicher Projektmitarbeiter am Institut für Politikwissenschaft der Universität Innsbruck sowie Lehrbeauftragter für verschiedene Fachbereiche und Studienprogramme der Universität Salzburg. Er forscht und publiziert zur Religionspolitologie sowie zu Themen der politischen Philosophie, der politischen Mythen und der politischen Ästhetik. 